\title{
A method for producing foveal retinal exposures in an awake, task-oriented, rhesus monkey*
}

\author{
DAVID O. ROBBINS ${ }^{\dagger}$ \\ Eye Research Foundation of Bethesda \\ Bethesda, Maryland 20014 \\ and

\section{HARRY ZWICK and GERALD C. HOLST Joint Laser Safety Team, Frankford Arsenal Philadelphia, Pennsylvania 19137}

A method for producing retinal exposures from a laser source in an awake, task-oriented $\mathbf{S}$ is presented. The technique allows for the measurement of rhesus monkey visual acuity immediately following exposures.

Functional studies concerned with the effects of intense irradiation on the visual process have been restricted to the evaluation of severe retinal morphological disruptions of the fovea in the rhesus (Tso \& Robbins, 1973; Weiskrantz \& Cowey, 1967; Yarczower, Walbarsht, Calloway, Fligsten, \& Malcolm, 1966; Graham \& Farrer, 1969). The effects of these foveal irradiation levels are usually permanent, producing impairment in visual acuity ranging from $40 \%$ to $80 \%$ of preexposure levels. Virtually no exploration of exposure levels at or below the transition from temporary to permanent visual losses has been conducted, since no technique is presently available to expose an awake, task-oriented animal. Immediate acuity effects following intense irradiation are critical in the exploration of thresholds for functional disruptions. In previous studies, anesthesia was required for placement of retinal lesions, thereby eliminating the possibility of immediate postexposure acuity measurements for at least $24 \mathrm{~h}$. The inability to measure transient changes in visual acuity at threshold and subthreshold power levels, as well as a means to follow the initial phases of deficits elicited by suprathreshold power levels, is a serious limitation in previous studies dealing with a functional approach to laser safety standards. In this paper, a method for producing foveal retinal exposures in an awake, task-oriented animal is presented along with a modification of a rapid method

*In conducting this research, the investigators adhered to the "Guide for Laboratory Animal Facilities and Care," as promulgated by the Committee on the Guide for Laboratory Animal Facilities and Care of the Institute of Laboratory Animal Resources, National Academy of Sciences-National Research Council. The authors are most grateful to Norman Krasnegor for suggesting the training paradigm and to Carl Richard Cavonius for his valuable suggestions throughout the course of this experiment.

+Present mailing address: Department of Psychology, Ohio Wesleyan University, Delaware, Ohio 43015 . to measure rhesus visual acuity (Graham, Farrer, Crook, $\&$ Garcia, 1970). This procedure can also be used to determine the immediate deficits in visual acuity and the temporal characteristics of the recovery process to irradiation at levels both above and below permanent functional damage.

Functional studies are important in determining the safety standards for laser irradiation, since morphological criteria alone tell little about the degradation of visual performance. The applicability of data from the rhesus as an experimental model for the development of laser safety standards is well substantiated by numerous studies demonstrating that the retinal physiology (Polyak, 1957) and visual performance (Weinstein \& Grether, 1940; Cowey \& Ellis, 1967; Cavonius \& Robbins, 1973; Schumake, Smith, \& Taylor, 1968) of rhesus and man are quite similar.

\section{METHODS}

\section{Subjects}

Two thesus monkeys (Macaca mulatta) served as Ss in the development of this procedure. The animals were housed and tested in modified primate chairs. Each rhesus was refracted by means of retinoscopy before testing, and no significant refractive errors were found.

\section{Apparatus}

Animals were tested for visual acuity in a light-tight, primate cubicle isolated from the programming equipment. A high-resolution rear-projection screen (Polacoat Co.) was mounted on the far wall of the cubicle and aligned with the S's line of regard. The screen subtended $3 \times 3$ deg at a distance of $1 \mathrm{~m}$ from the S's pupil. Background luminance of the screen was $2.5 \times 10^{2} \mathrm{~cd} / \mathrm{m}^{2}$, and its color temperature was $2,630^{\circ} \mathrm{K}$. The S's head was kept stationary during testing and exposure by four Plexiglas head restraints mounted on the primate chair, which prevented movement in any direction. An opaque facemask and a $7.0-\mathrm{mm}$ monocular iris diaphragm were aligned with the S's pupil and viewing screen so that eye position could be well controlled during testing. All testing was done with monocular viewing and natural pupils. Except for the screen, the test chamber was entirely dark.

\section{Discrimination Task}

The test patterns were conventional black Landolt rings presented against a light background. The rings were printed on Kodalith film (Eastman Kodak Co.), and when projected yielded a luminance contrast of approximately $97 \%$ between the rings and the light background. The thickness of the Landolt rings and the width of the gap that formed the critical detail were always $1 / 5$ of the diameter of the ring, and the size of the gap could be varied from 0.25 to $30 \mathrm{~min}$ of visual angle in equal steps. The test objects were presented in sets of four rings that were of equal diameter. Three of the rings in each set were gapless, while the fourth was a Landolt $C$ that appeared in a random position 


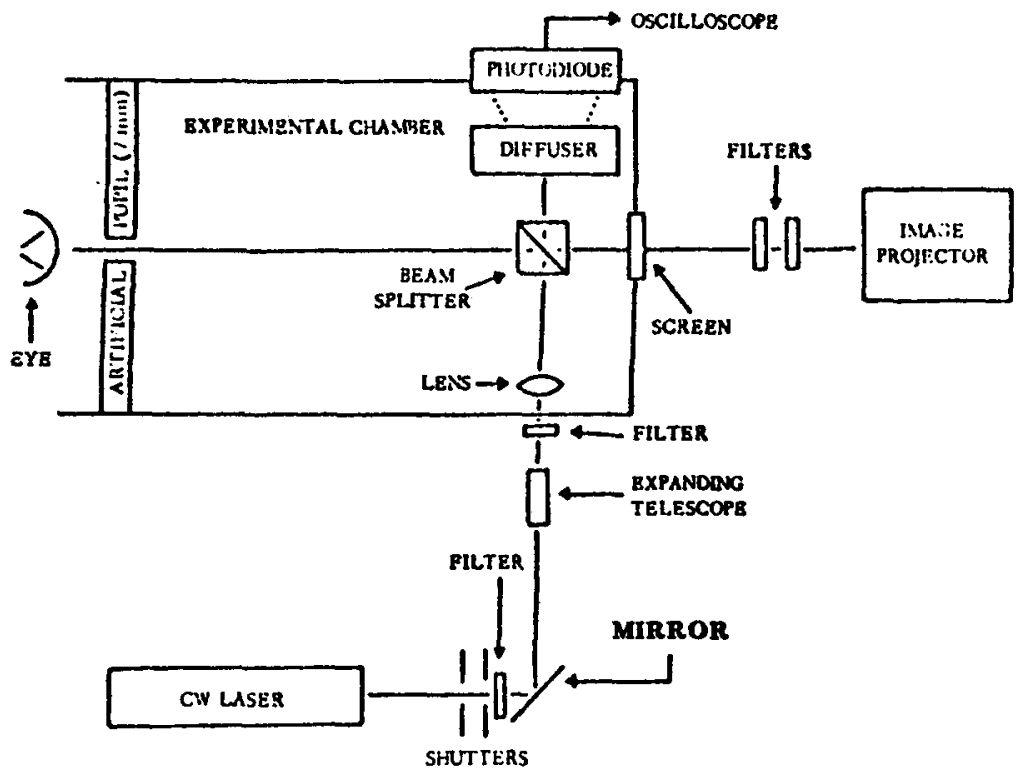

Fig. 1. Optical system used to produce an image of the laser source on the retina of the eye. The $\mathrm{CW}$ laser source used was a Spectra-Physics Model $125 \mathrm{He} \mathrm{Ne}$ laser, which had a maximum output power of $52 \mathrm{~mW}$. The various optical components used to produce a 150 -micron spot on the retina are explained in the text. This system produced a spot which had a maximum power of $21 \mathrm{~mW}$ at the cornea and a beam divergence of $0.01 \mathrm{rad}$.

within the set. Each ring was projected for $2 \mathrm{sec}$, and there was a $1-\mathrm{sec}$ dark interval between successive rings. No special cue was given when a set began; the stimuli appeared to the $\mathrm{O}$ as a single long series.

The Ss were trained, using negative reinforcement, to press a lever whenever a Landolt $C$ was presented and not to respond when gapless rings were presented. If the $S$ failed to respond to a Landolt $C$ during the 2 -sec presentation, he received a brief electric shock through an electrode in the seat of his Plexiglas primate chair. Shock was obtained from the secondary of a high-tension coil by discharging a capacitor into the primary; it was annoying but not highly painful or dangerous because of its short duration (Swinnen, Brady, \& Powell, 1969). To discourage the $S$ from responding indiscriminately to all rings, every third leverpress during gapless ring trials was also followed by shock. Training to threshold acuity levels took from 4 to 8 weeks.

Threshold acuity measurements were obtained by a tracking method (von Békésy, 1947), which allowed the $S$ to adjust in discrete steps the size of the test object about his own threshold. The size of the gap in the ring increased following incorrect $\mathrm{C}$ responses and decreased following correct $\mathrm{C}$ responses. Lever responses on gapless ring trials did not affect the size of the gap to be presented on the next set of trials. Means and standard deviations of the threshold visual acuity were obtained by use of Dixon \& Massey's (1957) statistics for the tracking method. During recovery, the average number of gapless rings relative to Landolt Cs was reduced to two in order to more quickly track transient changes in visual acuity as a function of time. Mean levels and variability were not affected by changing the ratio of Landolt $\mathrm{C}$ to gapless ring trials. Also unaffected was the number of lever responses for gapless ring trials, which were always very small (approximately 5\%) across the entire test session.

\section{Laser System}

A standard HeNe laser (Spectra Physics, Model 125A) served as the exposure source. A block diagram of the optical system is presented in Fig. 1. The entire laser system, with the exception of a focusing lens and beam splitter, was mounted outside the experimental chamber. The output beam diameter of the TEM mode laser at $632.8 \mathrm{~nm}$ was $2.0 \mathrm{~mm}$, with a beam divergence of 0.7 mrad. Output power at the source measured $52 \mathrm{~mW}$. The "raw" beam passed through a manual safety shutter and an electronic shutter. The electronic shutter (Danco Instruments) was driven by a Tektronix pulse form generator, and a calibrated pulse duration of $100 \mathrm{msec}$ was used in this study. The beam was attenuated by neutral density filters. The attenuated beam was diverted by a $4.5-\mathrm{cm}$-diam front surface mirror and entered a beam-expanding telescope, which produced a collimated beam of $8 \mathrm{~mm}\left(1 / \mathrm{e}^{2}\right.$ points $)$. The expanded beam passed into the experimental chamber and through a 1.25-diopter lens placed $85 \mathrm{~cm}$ in front of the animal's pupil. A $5 \times 10 \mathrm{~cm}$ coated beam splitter was placed $5 \mathrm{~cm}$ in front of the lens and at the intersection of the converging laser beam and beam from the Carousel projector. Mounted on the opposite side of the beam splitter was a diffuser and ultrafast photodiode (HPA 4203). The output of this detector was displayed on a memory oscilloscope and was calibrated against an EGG Model 580 radiometer placed at the corneal plane. The maximum power available at the cornea was $21 \mathrm{~mW}$. The power and pulse width of each irradiation was measured.

The laser flash was presented to the animal coaxial with a line between the artificial pupil and the gap in a specific Landolt ring subtending less than $1 \mathrm{~min}$ of arc. For determination of the line of sight, a 2-mm aperture was placed on the screen over the gap and a 4-mm aperture was placed at the plane of the cornea. A mirror, approximately $2 \mathrm{~m}$ behind the $4-\mathrm{mm}$ aperture was adjusted until it was normal to the line of sight. The beam splitter was then aligned such that the collimated beam from the laser (i.e., with the 1.25-diopter lens removed) passed through the $4-\mathrm{mm}$ aperture and reflected off the mirror back onto itself Coaxial alignment with the line of sight was verified by noting that the reflected beam also passed through the 2-mm aperture. The $80-\mathrm{cm}$ focal length lens was then positioned such that the cornea was in the focal plane of the lens so that changes in pupil diameter or small lateral movements of the S's head would not affect the amount of light entering the eye. The lens created a beam divergence of $0.01 \mathrm{rad}$. Assuming a focal length of $15 \mathrm{~mm}$ for the rhesus eye, the diameter of the beam on the retina was 150 microns.

\section{Laser Exposure}

Prior to laser exposure, stable acuity levels were established for each S. A criterion of, at minimum, 14 consecutive sessions of threshold measurements was used to establish a mean and standard deviation acuity level for each S. Prior to each exposure, a 15-min session was made and the mean for this preexposure testing was determined. The number of gapless ring trials was then reduced, and comparisons were made to assure a stable baseline. Failure of the $S$ to obtain mean acuity within one standard deviation of his predetermined acuity level on either schedule aborted the session. Session variability which exceeded baseline variability also aborted the session.

Exposures were made during threshold measurements after the above criteria were met. The $100-\mathrm{msec}$ flash was triggered by 
Fig. 2. Sample acuity data for one $S$ tested under maximum photopic conditions using a tracking technique. Horizontal pen excursions represent the presentation of Landolt $C$ trials, and the reciprocal visual angles that these gaps in Landolt rings subtended on the retina are shown in the left-hand scale. Corresponding Snellen notations for the various Landolt rings are shown on the right-hand scale. The solid line drawn through the data is the mean of the 20-min session, and the broken lines represent one standard deviation on either side of the mean.

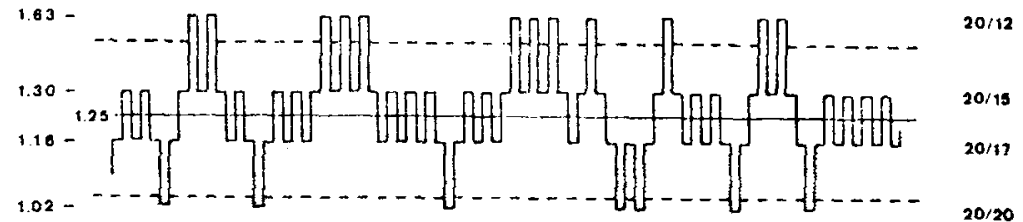

the animal's correct detection of his threshold Landolt ring, which corresponded to gap sizes of between 1.0 and 2.0 (min of arc $)^{-1}$. Exposures were made over power levels from 1.0 to $15 \mathrm{~mW}$ measured at the cornea, beginning with the lowest power level. No more than one exposure was made per session. Exposures were made only when the $\mathrm{S}$ made a correct detection of the Landolt $\mathrm{C}$ and only if the response was made during the first second of the trial.

Immediately after exposure, the recovery of acuity was measured until the $S$ returned to baseline. The session was terminated after a 15 -min stable baseline had been achieved. The entire test session lasted approximately $2 \mathrm{~h}$. Under conditions where total recovery was not complete within $2 \mathrm{~h}$, the session was still terminated and no exposure was made in the following or subsequent sessions until a standard baseline was again achieved.

\section{RESULTS}

Sample acuity for one $S$ is shown in Fig. 2. Acuity is defined in the usual manner as the reciprocal of the visual angle subtended by the gap at threshold. In this figure, reversals in the strip chart records were contingent upon the S's response on Landolt C trials. Correct detection of Landolt Cs caused the strip chart pen to plot upward and corresponded to the presentation of smaller ring diameters. Incorrect detection of Landolt Cs caused the strip chart pen to plot downward and corresponded to the presentation of larger ring diameters. Horizontal pen excursions represent Landolt $C$ trials. The reciprocal visual angle that the gap in the Landolt ring subtended on the retina is shown on the left-hand ordinate with corresponding Snellen notations on the right-hand ordinate. Mean acuity of 1.25 ( $\mathrm{min}$ of $\mathrm{arc}^{-1}$ was obtained in this session and is represented by the solid line drawn through the data. The broken lines represent one standard deviation $(\sigma=0.13)$ on either side of the mean. Mean acuity of this $S$ derived from several weeks of threshold testing was 1.12 (min of arc) $)^{-1}$.

Recovery from a $7-\mathrm{mW}$ exposure is shown in Fig. 3 for the same $S$ shown in Fig. 2. Time, relative to exposure, is indicated on the abscissa. In the left-hand portion of the figure, acuity is shown for a $15-\mathrm{min}$ period preceding laser exposure, using a 4:1 ratio of gapless rings to Landolt Cs. Preexposure mean acuity of 1.25 (min of arc) ${ }^{-1}$ was within one standard deviation of this S's mean baseline acuity of 1.12 (min of arc) ${ }^{-1}$. Preceding the exposure, the $S$ was transferred to a $2: 1$ ratio of gapless rings to Landolt $\mathrm{Cs}$ and tested for
Fig. 3. Sample data of threshold acuity using a tracking technique prior to and immediately following laser irradiation. In this session, the $S$ was exposed to a $7 \cdot \mathrm{mW}$ laser flash of $100 \mathrm{msec}$ duration. The occurrence of the exposure is indicated in the figure by an arrow, and corresponds to the zero point on the abscissa. The ordinate indicates the various sizes of the gaps in presented Landolt rings and is plotted in reciprocal visual minutes of arc. Acuity as defined by this scale is greatest at $1.63 \mathrm{~min}$ of $\operatorname{arc})^{-1}$ and least at $0.47(\mathrm{~min} \text { of } \operatorname{arc})^{-1}$. This scale is measured in discrete steps, since the vertical excursions of the strip chart recorder were taken from a nonlinear potentiometer mounted on the slide tray and recorded only tray position. The abscissa represents the presentation of the Landolt Cs, and corresponding times (in minutes) for representative trials are indicated relative to exposure. The break in the records at $-2 \mathrm{~min}$ and again at $14 \mathrm{~min}$ is the point at which the ratio of completed ring trials to Landolt $C$ trials was shifted.

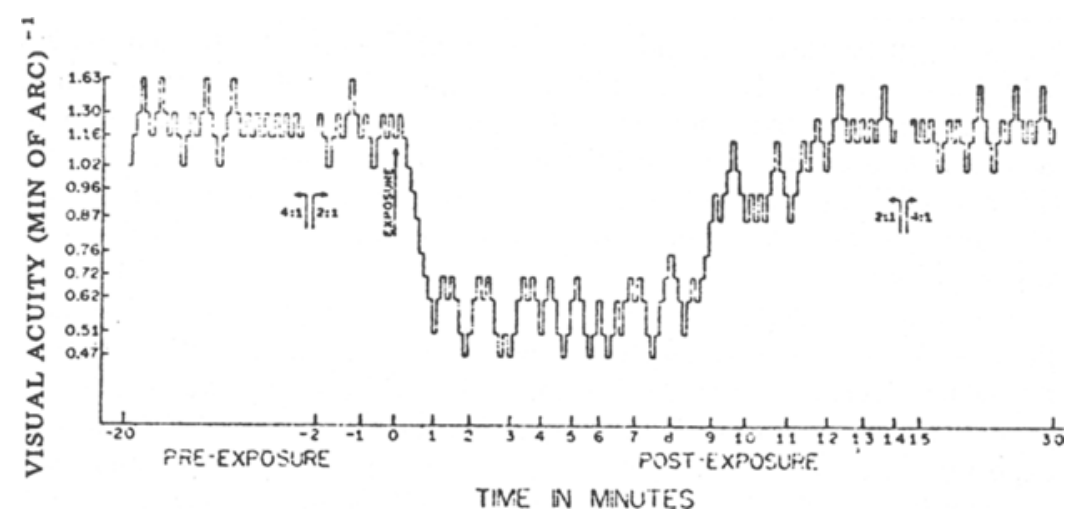




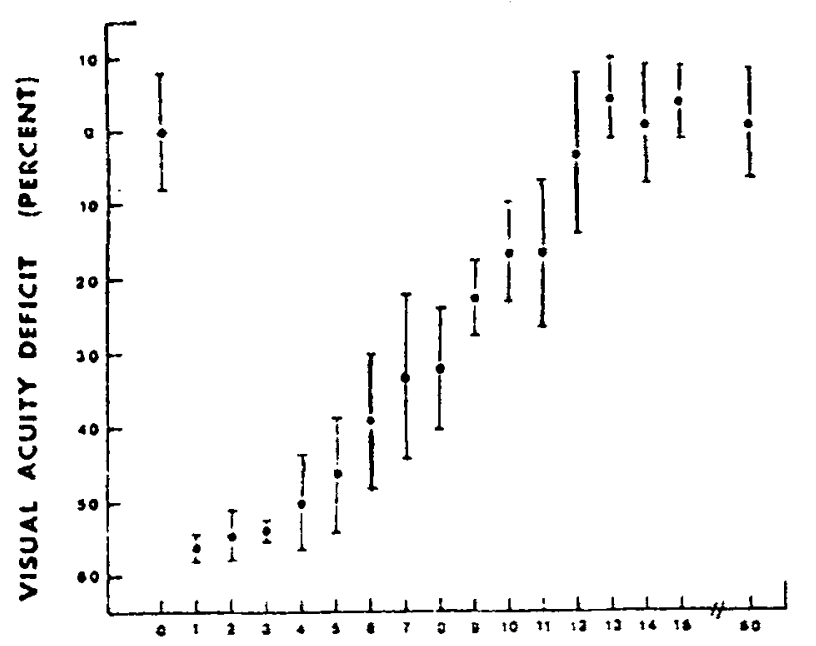

MINUTES AFTER EXPOSURE

Fig. 4. Percent deficit in visual acuity as a function of time following a $5.7-\mathrm{mW}$ laser exposure. Visual deficits were calculated from mean acuities (min of arc) ${ }^{-1}$ of consecutive postexposure intervals relative to the mean preexposure acuity ( $\min$ of arc) ${ }^{-1}$. The exposure was $100 \mathrm{msec}$ in duration and presented immediately following a correct detection of a 1.16 ( $\mathrm{min}$ of $\operatorname{arc})^{-1}$ Landolt $\mathrm{C}$. Individual data points represent the mean of four exposure sessions for each running minute, and the vertical lines indicate one standard deviation on either side of the mean. Zero marked on the time abscissa is the S's acuity level immediately preceding exposure.

approximately $2 \mathrm{~min}$. No shift in acuity occurred following this reduction in the number of gapless ring trials. In this session, the $S$ was exposed following a correct detection of a 1.16 ( $\mathrm{min}$ of arc) ${ }^{-1}$ Landolt $\mathrm{C}$ and corresponded to the zero point on the abscissa of this figure. Immediately after exposure, the S's acuity decreased to 0.51 ( $\mathrm{min}$ of $\mathrm{arc})^{-1}$, which corresponds to an acuity deficit of $59 \%$ relative to preexposure acuity. This visual deficit lasted 9 min before acuity gradually returned to the S's mean preexposure acuity level. Total recovery from the initial deficit was complete within approximately $13 \mathrm{~min}$. Threshold testing using the $2: 1$ ratio of gapless rings to Landolt $\mathrm{Cs}$ was continued for 3 additional minutes. The ratio of gapless rings to Landolt Cs was then shifted back to $4: 1$, and postexposure acuity measurements were extended for an additional $15 \mathrm{~min}$. This is shown in the extreme right-hand portion of this figure. No significant shifts in pre- and postexposure acuity were found.

The percent deficit in visual acuity relative to preexposure acuity was plotted as a function of time following laser exposure for various power levels. A mean recovery function for a single $S$ based on four exposure sessions is shown in Fig. 4. The exposure power was $5.7 \mathrm{~mW}$. The mean acuity level and standard deviation for each running minute following exposure are shown. In this figure, the S's mean acuity immediately decreased to 0.55 ( $\mathrm{min}$ of arc) ${ }^{-1}$ following exposure and is comparable to the maximum deficits elicited by other exposure powers (see Fig. 3). Initial deficits in acuity were independent of power level over a range of 1 log unit below levels that produced permanent functional deficits (Robbins, Zwick, \& Holst, 1973). These acuity deficits ranged from 0.59 to 0.44 (min of arc) $)^{-1}$, which corresponds to acuity deficits of $40 \%-60 \%$. Both the duration of the maximum deficit and the total recovery time, however, were directly related to exposure power. In this figure, recovery began almost immediately following exposure and the S's acuity increased monotonically over the entire duration of the recovery period. Total recovery was complete within $12 \mathrm{~min}$ of exposure. With more intense exposure powers, recovery often did not begin until several minutes had elapsed and the recovery process encompassed a longer period of time. In Fig. 3, for example, the initial deficit lasted approximately $9 \mathrm{~min}$.

\section{DISCUSSION}

A method for producing retinal exposures from a laser source in an awake, task-oriented $\mathrm{S}$ was presented. This technique allowed for the measurement of rhesus visual acuity immediately following exposure and can be used to derive the power levels required for the transition from temporary to permanent functional deficits. It differs from previous methods where anesthesia was required for placement of retinal lesions.

Visual acuity was obtained by a tracking method, using negative reinforcement, which allowed for immediate measurements of transient shifts in acuity as well as the temporal course of recovery within a single test session. Contrary to the results of Graham, Farrar, Crook, \& Garcia (1970), aversive conditioning was successfully used in both the initial and testing stages of this procedure. The initial training phases were also considerably . shorter than the 6 months stated by Graham et al (1970). However, the discrimination procedure used by Graham et al (1970) involved two response levers as compared to the single response lever used in the present experiment. In this experiment, little or no lever responding occurred to completed rings in trained Ss regardless of acuity level. Stable acuity levels were obtained within and across sessions, and mean acuity derived by this procedure compare well with other studies (Weinstein \& Grether, 1940; Cowey \& Ellis, 1967; Cavonius \& Robbins, 1973).

Exposure to the laser flash produced an immediate, but temporary, deficit in acuity. The magnitude of the maximum acuity deficit in this experiment is consistent with those studies where permanent foveal disruption was produced (Yarczower et al, 1966; Tso \& Robbins, 1973) and suggests foveal involvement. This deficit is consistent with the manner in which exposures were initiated, that is, during measurements of maximal acuity under photopic conditions where involvement of the centralmost area of the retina was required. Our results imply that in this experiment the Ss maintained fixation on the discriminanda immediately following their overt behavioral response in the vast majority of 
trials. In a few cases, laser exposures elicited little or no acuity deficits, and in some cases it might be suggested that eye movements or lid closures resulted in either irradiation of extrafoveal areas or reduced foveal involvement. In other cases, where exposures elicited no significant acuity deficits, the alignment of the beam with the discriminanda had been in error by as little as $0.5 \mathrm{deg}$. If nonfoveal areas were irradiated, little shift in maximal acuity should be observed, since only foveal areas are involved in such minute critical detail.

Repeated exposures at the same power level elicited similar functional deficits, which implies that irradiation effects were of a physiological rather than of a behavioral origin. Behavioral disruptions as a result of sudden distracting light flashes irradiating retinal areas would most likely produce erratic performance rather than the consistent and systematic acuity shifts noted in this experiment. Preliminary results of the effects of power level on acuity, for example, suggest that the duration of the maximum deficit and time required for total recovery increased systematically with exposure power. The fact that exposures to intense irradiation did not result in total functional impairment also implies a physiological component and could be explained by at least two different hypotheses. First, the exposure of foveal areas could result in an incomplete saturation of the photoreceptors and the resultant acuity levels obtained represent the activity of nondepleted foveal photoreceptors. Recovery would then represent the time required for affected foveal photoreceptors to again become functional. An alternate hypothesis is that the intense levels of exposures employed in the present study resulted in complete disruption of function in the retinal area exposed. The lack of total functional impairment would then imply the use of alternate retinal areas to make the required discrimination. If this hypothesis were true, the maximum deficit elicited would then correspond to the sensitivity of the surrounding areas outside the fovea which were unaffected by the exposure. The acuity levels obtained immediately following exposure correspond well with parafoveal acuity in rhesus (Yarczower et al 1966; Tso \& Robbins, 1973) and could support this hypothesis. In addition, the independence in the magnitude of the initial deficit with changes in exposure power suggests total involvement of specific retinal areas. Less than total receptor depletion should be represented by a graduation of acuity deficits as exposure power varied. The improvement of acuity with time could represent functional recover ${ }^{\prime}$ of foveal areas as Ss continually scanned the test target following exposure to bring into play their more sensitive retinal area.

One possible means of differentiating the two hypotheses would be to employ a larger spot, where energy density is the same as used in this study. If parafoveal areas are being used to make the discrimination, as the second hypothesis suggests, one would expect that increased spot size would increase the magnitude of the maximum deficit. At the present time, we are exploring this aspect.

\section{REFERENCES}

Békésy, G. von. A new audiometer. Acta Oto-laryngologica, 1947, 35, 411-422.

Cavonius, C. R., \& Robbins, D. O. Relationship between luminance and visual acuity in the rhesus monkey. Journal of Physiology (London), 1973, in press.

Cowey, A., \& Ellis, C. A. Visual acuity of rhesus and squirrel monkeys. Journal of Comparative \& Physiological Psychology. $1967,64,80-84$

Dixon, W. J., \& Massey, F. J. Introduction to statistical analysis. New York: McGraw-Hill, 1957.

Graham, E. S., \& Farrer, D. N. Retinal lesions and visual acuity in the rhesus monkey (Macaca mulatta). Report No. ARL-TR-69-3, Aerospace Medical Division, Air Force Systems Command, Holloman AFB, New Mexico, 1969.

Graham, E. S. Farrer, D. N. Crook, G. H. \& Garcia, P. V. A self-adjustment procedure for measuring the visual acuity of rhesus monkeys. Behavior Research Methods \& Instrumentation, 1970, 2, 301-305.

Polyak, S. The vertebrate visual system. Chicago: University of Chicago Press, 1957.

Robbins, D. O., Zwick, H., \& Holst, G. C. Symposium on recent advances in color vision deficiencies. In Modern problems in ophthalmology. New York: Karger, 1973 (in press).

Schumake, S. A., Smith, J. C., \& Taylor, H. L. Critical flicker fusion in rhesus monkeys. Psychological Record, 1968, 8, $537-542$.

Swinnen, M. E. T., Brady, J. V. \& Powell, M. G. A new device for the application of shock. Behavior Research Methods \& Instrumentation, $1969,1,184$.

Tso, M. O. M., \& Robbins, D. O. Photic maculopathy: A study of functional and pathologic correlation. Proceedings of Gonin Society, 1973.

Weiskrant2, L., \& Cowey, A. Comparison of the effects of striate cortex and retinal lesions on visual acuity in the monkey. Science, 1967, 155, 104-106.

Weinstein, B., \& Grether, W. A comparison of visual acuity in the rhesus monkey and man. Journal of Comparative Psychology, $1940,30,187-195$.

Yarczower, M., Wolbarsht, M. L. Galloway, W. D., Fligsten, K. E., \& Malcolm, R. Foveal function in monkeys. Science, 1966 , $152,1392-1393$

(Received for publication July 16,1973 ; revision received August 30,1973.) 